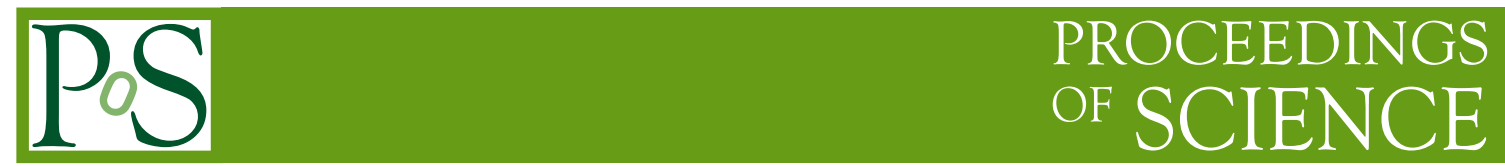

\title{
Higgs search by CMS
}

\section{Artur Kalinowski ${ }^{* \dagger}$}

Faculty of Physics, University of Warsaw, Hoża 69, 00-681 Warsaw, Poland

E-mail: Artur.Kalinowskiecern.ch

We present results of the Higgs boson searches at the CMS experiment with up to $1.7 \mathrm{fb}^{-1}$. We describe the analyses in the main search channels. Since no excess over the expected Standard Model background was observed we present 95\% CL exclusion limits on the Higgs boson mass.

LHC on the March,

November 16-18, 2011

Protvino, Moscow region, Russian Federation

* Speaker.

On behalf of the CMS Collaboration 

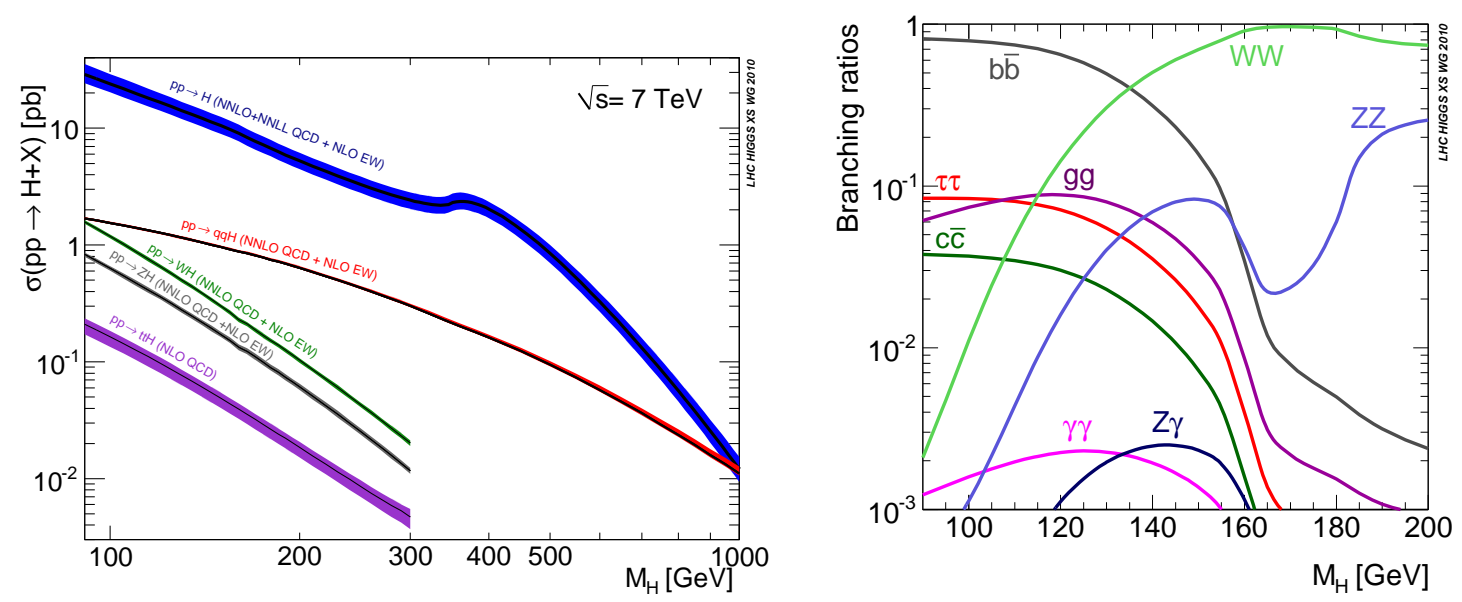

Figure 1: Standard Model Higgs boson production cross sections for $7 \mathrm{TeV}$ pp collisions (left), and decay branching ratios (right) [6].

\section{Introduction}

Since the Higgs boson is the last missing piece of the Standard Model it is being searched for at least last three decades in many experiments. The direct lower limit on the Higgs boson mass comes from electron-positron collision data at LEP, and is $\mathrm{m}_{\mathrm{H}}>114.4 \mathrm{GeV} / \mathrm{c}^{2}$ at $95 \% \mathrm{CL}$ [1]. Recent results from Tevatron with up to $8.6 \mathrm{fb}^{-1}$ of $\mathrm{p} \overline{\mathrm{p}}$ data exclude also a mass window of $156<\mathrm{m}_{\mathrm{H}}<177 \mathrm{GeV} / \mathrm{c}^{2}$ [2]. The Higgs boson search, within both Standard Model and its extensions, is also in the centre of the CMS experiment physics program [3]. The CMS Collaboration pursued Higgs boson search in total of 27(24) sub-channels in the low (high) mass region. In this contribution we briefly describe the searches in most important Standard Model channels, and present the exclusion limits which significantly extend limits obtained in 2010 [4, 5]. We also present limits from combination of all the search channels used at the CMS to the moment.

Possible search for new particles strategies are driven by the available production and decay modes of particle in question. The main modes for the Standard Model Higgs production at the proton-proton collider are gluon-gluon fusion, yielding cross sections of order of 1 to $10 \mathrm{pb}$ for Higgs masses of $400 \mathrm{GeV} / \mathrm{c}^{2}$, and $110 \mathrm{GeV} / \mathrm{c}^{2}$ at NNLO+NNLL QCD+NLO EW calculation order, Figure 1(left) [6]. The main decay paths are $b \bar{b}$ and $\tau \tau$ below threshold for decay to vector boson pair, and WW and ZZ above, as presented on Figure 1(right) [6].

\section{Searches in $\mathrm{H} \rightarrow \gamma \gamma$ final state}

The Higgs branching ratio to $\gamma \gamma$ is extremely small, at the level of $0.1 \%$, but with excellent energy resolution of the CMS electromagnetic calorimeter one can reach resolution of two photons invariant mass of order of $0.7 \%$ making the searches in this channel viable.

The background for this final state are di-photon production, and $\gamma+$ jet, or jet+jet, where one of QCD jets could be be misidentified as a photon in the event reconstruction. The event selection includes photon isolation, and categorisation of events depending on the photon reconstruction quality, best in the barrel region of the detector, worst in the endcaps. 

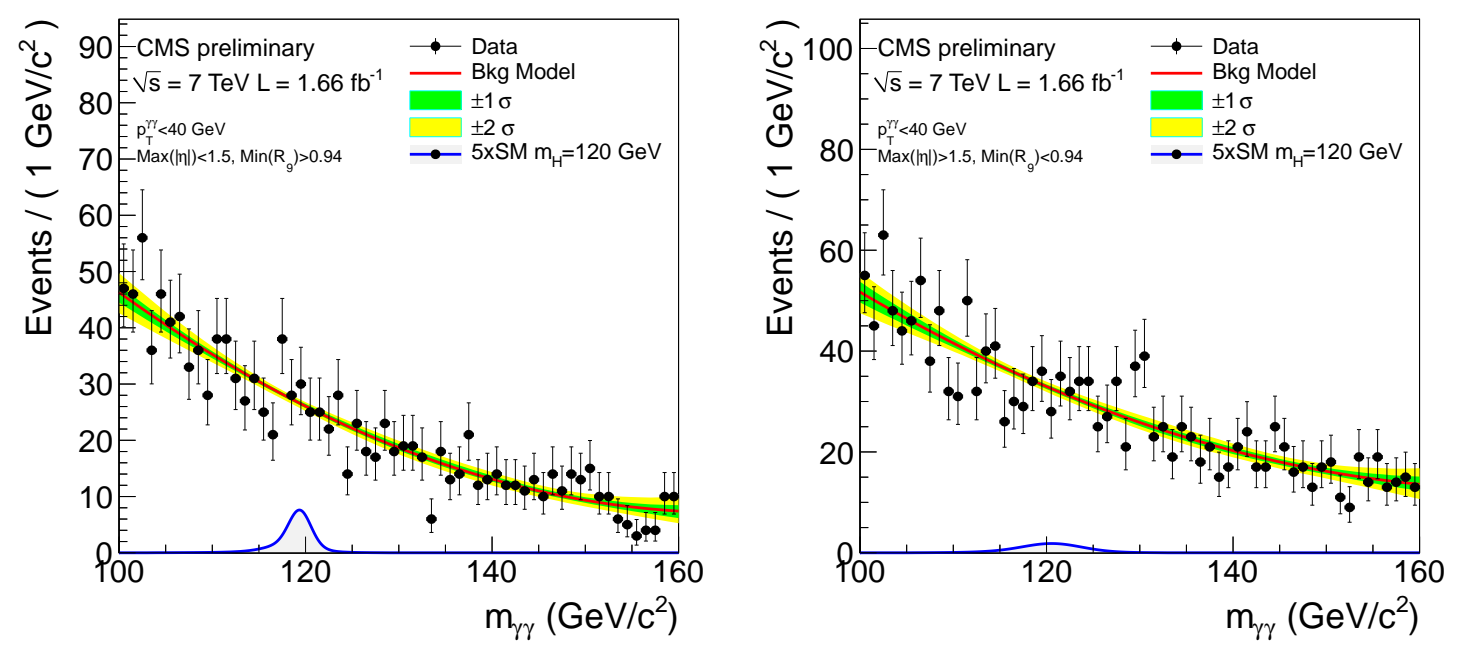

Figure 2: Observed two photon invariant mass distribution with background model fits overlaid.Figure on the left presents the photons in the best category: both photons in the ECAL barrel region, with a narrow shower, figure on the right presents the photons in the worst category: both photons in the ECAL endcap region, with a wide reconstructed shower. Expected signal contribution for $\mathrm{m}_{\mathrm{H}}=120 \mathrm{GeV} / \mathrm{c}^{2}$ with signal cross section multiplied by factor of 5 is shown.

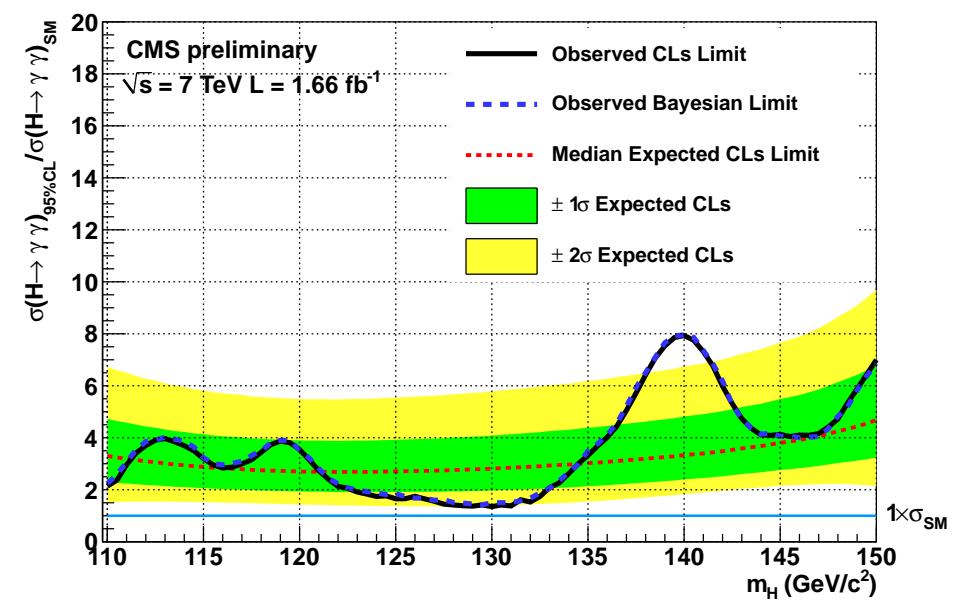

Figure 3: Exclusion limit on cross section of a SM Higgs boson $\mathrm{H} \rightarrow \gamma \gamma$ decay relative to the SM cross section, as a function of the boson mass obtained with $1.66 \mathrm{fb}^{-1}$ of data collected at $\sqrt{\mathrm{s}}=7 \mathrm{TeV}$.

Figure 2 presents the observed di-photon invariant mass distribution in best (left) and worse (right) photon category. A data driven background model is overlaid, and signal contribution multiplied by factor of 10 with respect to the Standard Model expectation is presented.

Figure 3 presents the expected 95\% CL exclusion limit as a function of the Higgs mass. The expected exclusion limit at 95\% CL is between 2.7 and 4.7 times the Standard Model cross section, and the observed limit fluctuates between about 1.3 and 8 times the Standard Model cross section. 

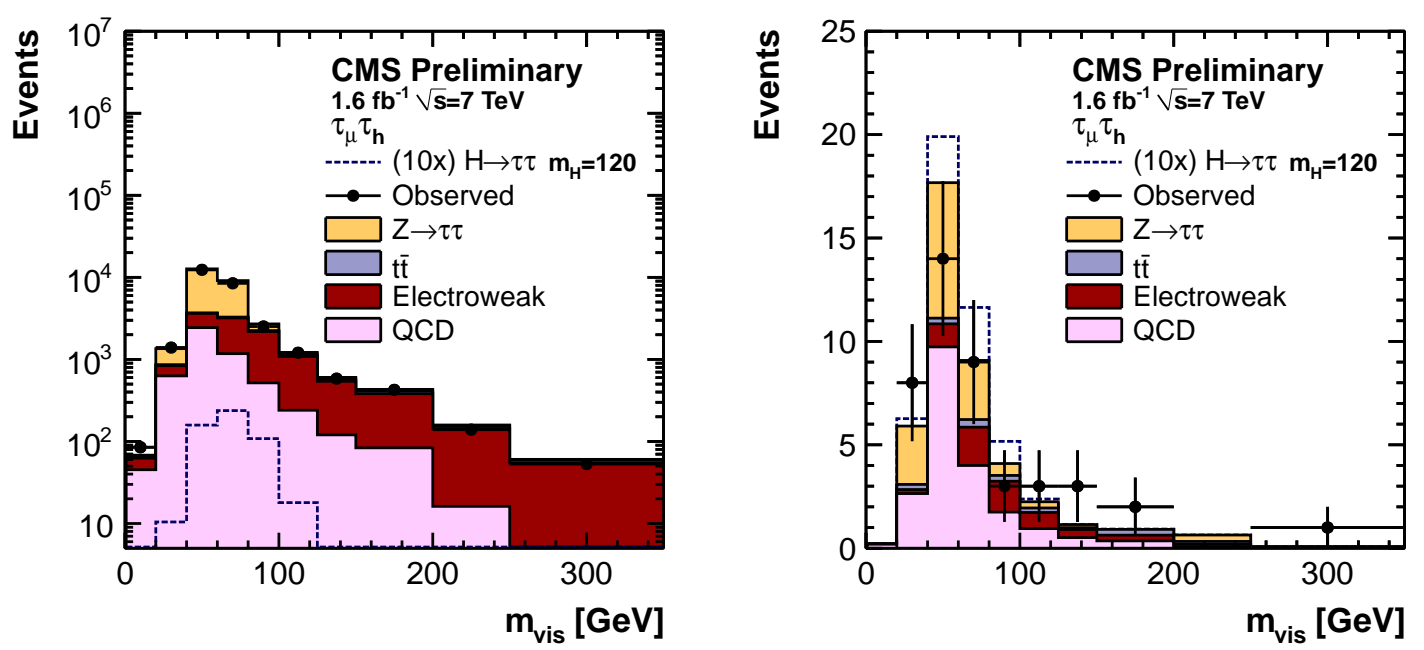

Figure 4: Observed distribution of visible mass of $\tau \tau$ system in the $\mu+\tau_{\text {jet }}$ final state after inclusive (left), and VBF (right) selections. Expected signal contribution for $\mathrm{m}_{\mathrm{H}}=120 \mathrm{GeV} / \mathrm{c}^{2}$ with signal cross section multiplied by factor of 10 is shown.

\section{Searches in $H \rightarrow \tau \tau$ final state}

In the CMS searches in $\mathrm{H} \rightarrow \tau \tau$ were made for three decay channels of the $\tau \tau$ pair: one $\tau$ decaying into electron or muon, and the other one decaying hadronically, denoted as $\tau_{\text {had }}$, and with both $\tau$ s decaying leptonically into leptons of different flavour. Analysis in each of above channels was divided into two exclusive categories: inclusive - aiming at $\mathrm{gg} \rightarrow \mathrm{H}$ production mode, and $\mathrm{VBF}$ aiming at $\mathrm{qq} \rightarrow \mathrm{qqH}$. The signal signatures are isolated lepton from semileptonic tau decay and isolated tau-like jet in case one $\tau$ decays hadronically. The most challenging background is the irreducible $\mathrm{Z} \rightarrow \tau \tau$, most important for the low mass region. Other background sources are $\mathrm{QCD}, \mathrm{tt}, \mathrm{W}+\mathrm{jets}, \mathrm{Z} \rightarrow$ ee and $\mathrm{Z} \rightarrow \mu \mu$, The event selection includes lepton isolation, hadronic tau identification and cut on the $\mathrm{m}_{\mathrm{T}}\left(1, \mathrm{E}_{\mathrm{T}}^{\mathrm{miss}}\right)$ to reduce the $\mathrm{W}$ background. In the VBF selection there are two additional jets required with $\mathrm{E}_{\mathrm{T}}>30 \mathrm{GeV}$. Following kinematic properties of the VBF production the jets are required to lie in the opposite parts of the detector with $\eta^{\text {jet } 1} \cdot \eta^{\text {jet } 2}<0$, to have large separation in the pseudorapidity: $\left|\eta^{\text {jet } 1}-\eta^{\text {jet } 2}\right|>3.2$, and to form high invariant mass system with $\mathrm{m}($ jet 1 , jet 2$)>350 \mathrm{GeV}$. Figure 4 presents observed visible mass of two tau leptons in the inclusive (left), and in the VBF (right) categories.

Figure 5 presents the expected $95 \%$ CL exclusion limit as a function of the Higgs mass. The expected exclusion limit at 95\% CL is between 5.4 and 12.5 times the Standard Model cross section, and the observed limit fluctuates between about 6 and 17.9 times the Standard Model cross section.

\section{Searches in $\mathrm{H} \rightarrow \mathrm{b} \bar{b}$ final state}

The $b \bar{b}$ decay mode is dominant for low mass SM Higgs boson, but this is a very challenging channel due to overwhelming background from QCD b $\bar{b}$ production. In CMS the $b \bar{b}$ decay mode 


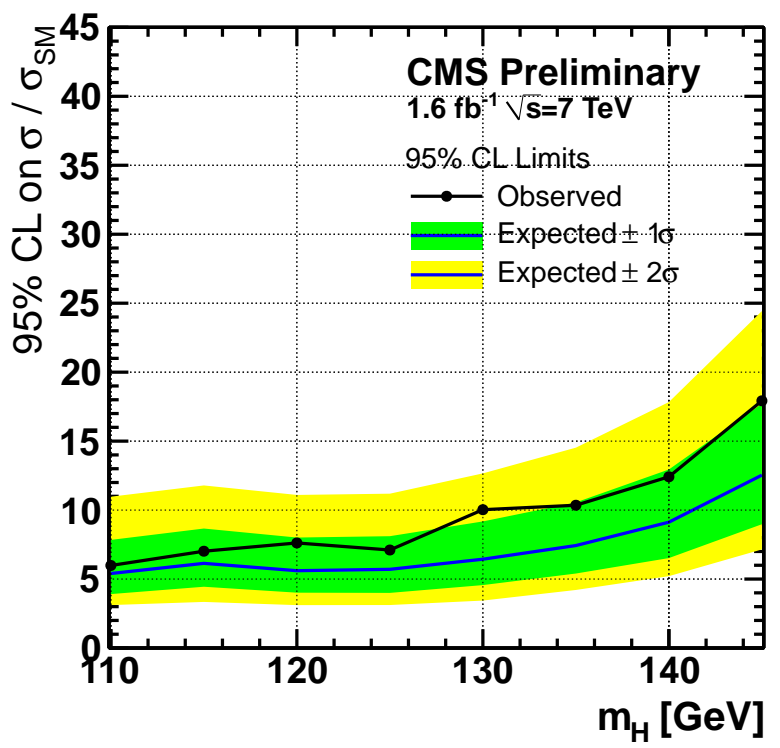

Figure 5: Exclusion limit on cross section of a SM Higgs boson $\mathrm{H} \rightarrow \tau \tau$ decay relative to the SM cross section, as a function of the boson mass, obtained with $1.66 \mathrm{fb}^{-1}$ of data collected at $\sqrt{\mathrm{s}}=7 \mathrm{TeV}$. All analysed $\tau \tau$ decay modes, and inclusive and VBF categories are combined.
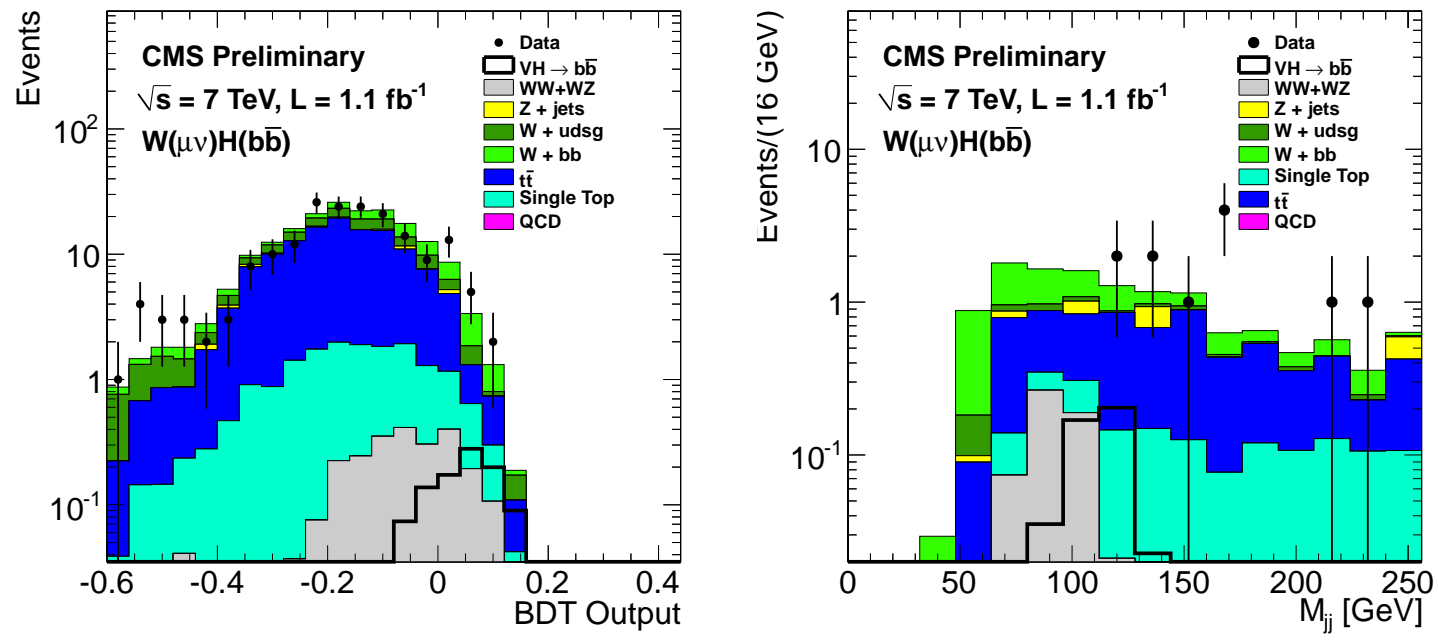

Figure 6: Observed distributions of DBT output (left) and $M_{j j}$ (right) in the $H \rightarrow b \bar{b}$ analysis. Expected signal contribution for $\mathrm{m}_{\mathrm{H}}=115 \mathrm{GeV} / \mathrm{c}^{2}$ with signal cross section multiplied by factor of 10 is shown.

is studied for the $\mathrm{VH}$ associated production mode, where associated vector boson provides additional handle to reduce the background contribution. The following modes are studied: $\mathrm{W}(\mu v) \mathrm{H}$, $\mathrm{W}(\mathrm{e} v) \mathrm{H}, \mathrm{Z}(\mu \mu) \mathrm{H}, \mathrm{Z}(\mathrm{ee}) \mathrm{H}$ and $\mathrm{Z}(v v) \mathrm{H}$. The event selection includes requirements for b-tagged jets, $\mathrm{E}_{\mathrm{T}}^{\mathrm{miss}}$ quality, and high $\mathrm{p}_{\mathrm{T}}$ of associated bosons. The analysis is made using standard cut based selections, and also with multivariate Boosted Decision Tree (BDT) method.

Figure 6(left) presents the observed distribution for the BDT output, and Figure 6(right) presents 


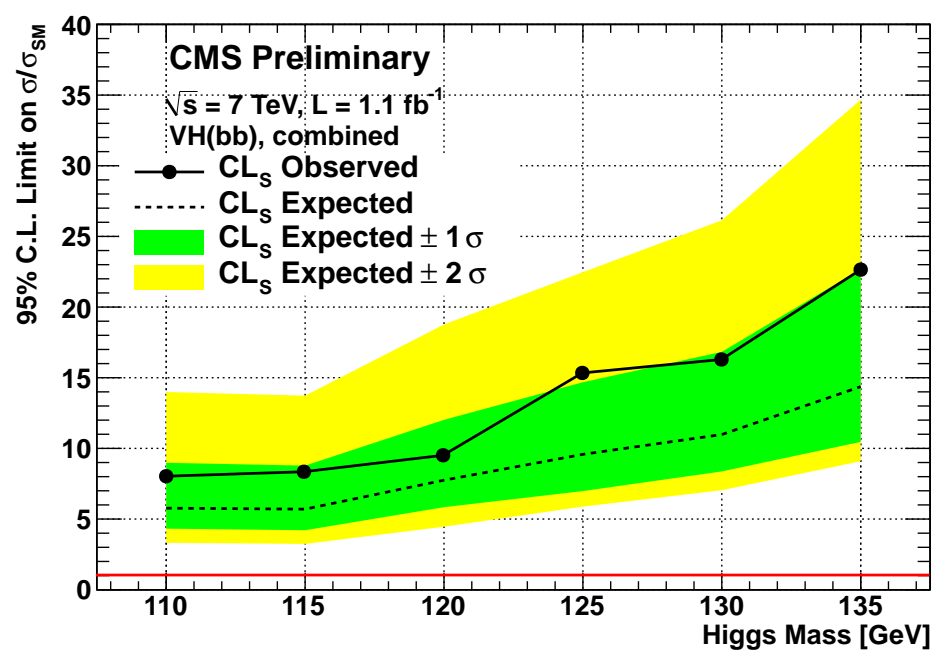

Figure 7: Exclusion limit on cross section of a SM Higgs boson $\mathrm{H} \rightarrow \mathrm{b} \overline{\mathrm{b}}$ decay relative to the SM cross section, as a function of the boson mass obtained with $1.1 \mathrm{fb}^{-1}$ of data collected at $\sqrt{\mathrm{s}}=7 \mathrm{TeV}$.

observed distribution of the two, $b$-jets invariant mass, both with expected signal contribution for $\mathrm{m}_{\mathrm{H}}=115 \mathrm{GeV} / \mathrm{c}^{2}$ overlaid.

Figure 7 presents the expected 95\% CL exclusion limit as a function of the Higgs mass. The expected exclusion limit at 95\% CL is between 5.7 and 14.4 times the Standard Model cross section, and the observed limit fluctuates between about 7.96 and 22.6 times the Standard Model cross section.

\section{Searches in $\mathrm{H} \rightarrow \mathrm{WW}$ final state}

The best search channel for $\mathrm{H} \rightarrow \mathrm{WW}$ is the case when both $\mathrm{W}$ bosons decay leptonically, providing excellent handle to select the signal events. The $\mathrm{H} \rightarrow \mathrm{WW} \rightarrow 2 \mathrm{l}+2 v$ final state signatures are two isolated high $\mathrm{p}_{\mathrm{T}}$ leptons coming from $\mathrm{W}$ decays, large $\mathrm{E}_{\mathrm{T}}^{\text {miss }}$ due to the two neutrinos in the final state and low jet activity in the event. As WW bosons pair comes from spin 0 particle decay, specific lepton azimuthal correlations due to angular momentum conservation can be used to select the signal events. The main reducible background contribution comes from Drell-Yan lepton production, $\mathrm{t} \overline{\mathrm{t}}, \mathrm{W}+\mathrm{t}, \mathrm{W}+\mathrm{jets}, \mathrm{WZ}$ and $\mathrm{ZZ}$. The genuine $\mathrm{WW}$ pair production is the irreducible background. The analysis selection is based on a classical cut based approach, or a neural network (NN) output. Figure 8 presents examples of variables used for the cut based selection: the azimuthal separation between the two leptons (left), and their invariant mass (right).

Figure 9 presents the expected 95\% CL exclusion limit as a function of the Higgs mass. The expected exclusion limit at 95\% CL is between 0.28 and 7.7 times the Standard Model cross section, and the observed limit fluctuates between about 0.5 and 6.3 times the Standard Model cross section. The observed limit allows for 95\% CL exclusion of SM Higgs boson in a $147-194 \mathrm{GeV} / \mathrm{c}^{2}$ mass window. 

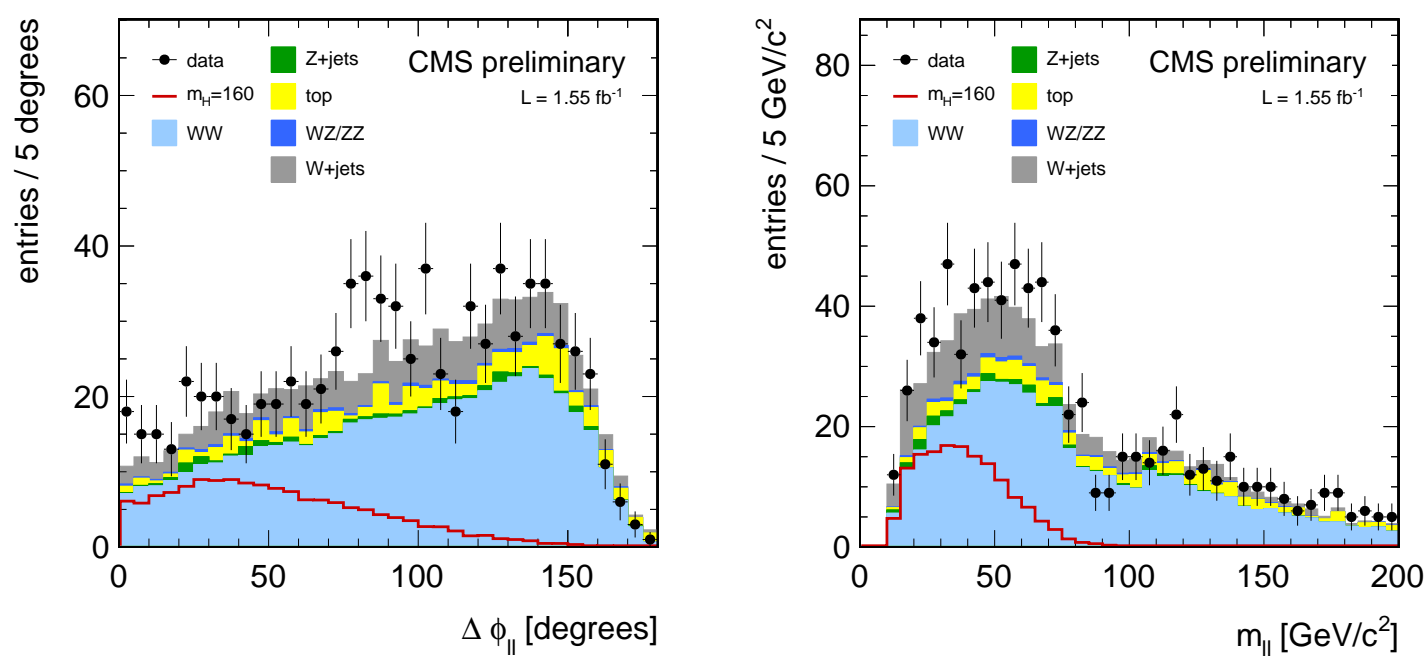

Figure 8: Observed distribution of the azimuthal separation, $\Delta \Phi_{11}$ (left), and invariant mass of two leptons (right). Expected signal $\mathrm{H} \rightarrow \mathrm{WW}$ signal with $\mathrm{m}_{\mathrm{H}}=160 \mathrm{GeV} / \mathrm{c}^{2}$ is shown.

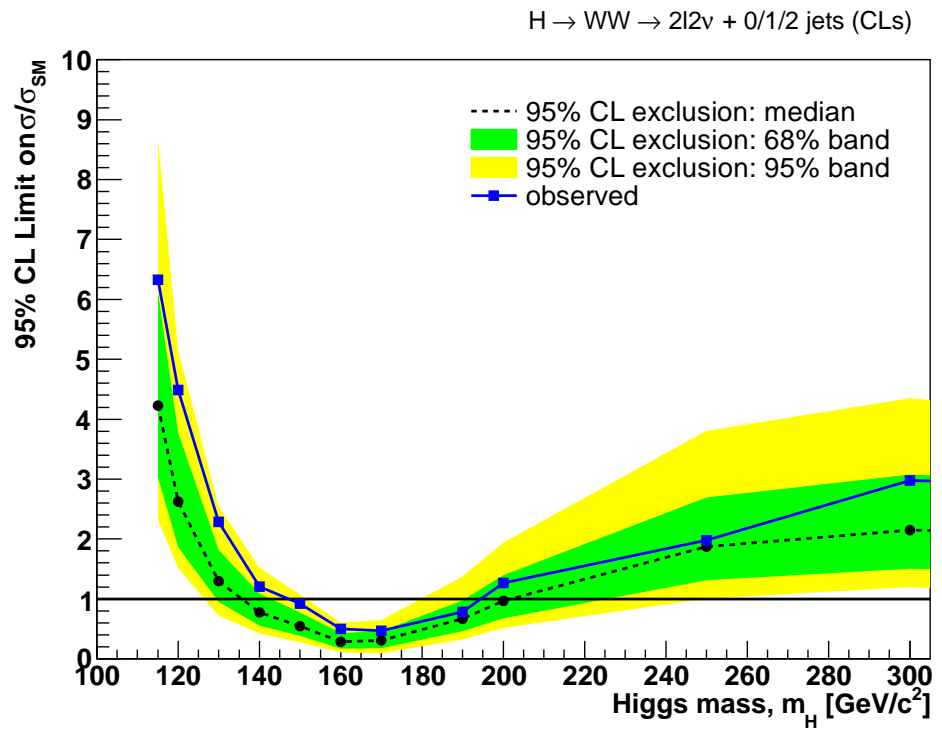

Figure 9: Exclusion limit on cross section of a SM Higgs boson $\mathrm{H} \rightarrow \mathrm{WW} \rightarrow 212 v$ decay relative to the SM cross section, as a function of the boson mass obtained with $1.55 \mathrm{fb}^{-1}$ of data collected at $\sqrt{\mathrm{s}}=7 \mathrm{TeV}$.

\section{Searches in $\mathrm{H} \rightarrow \mathrm{ZZ}$ final state}

The most power full SM Higgs search mode is search in $\mathrm{H} \rightarrow \mathrm{ZZ}$ decay with subsequent $\mathrm{Z} \rightarrow \mathrm{ll}$ decay. The selection in this channel includes requirement for at least two isolated leptons and moderate $\mathrm{Z}$ mass constraint $\left(60<\mathrm{m}_{11}<120 \mathrm{GeV} / \mathrm{c}^{2}\right)$ for one lepton pair, allowing other to come from off-shell $\mathrm{Z}$ with loose mass constraint $\left(20<\mathrm{m}_{11}<120 \mathrm{GeV} / \mathrm{c}^{2}\right)$. The main background is the 

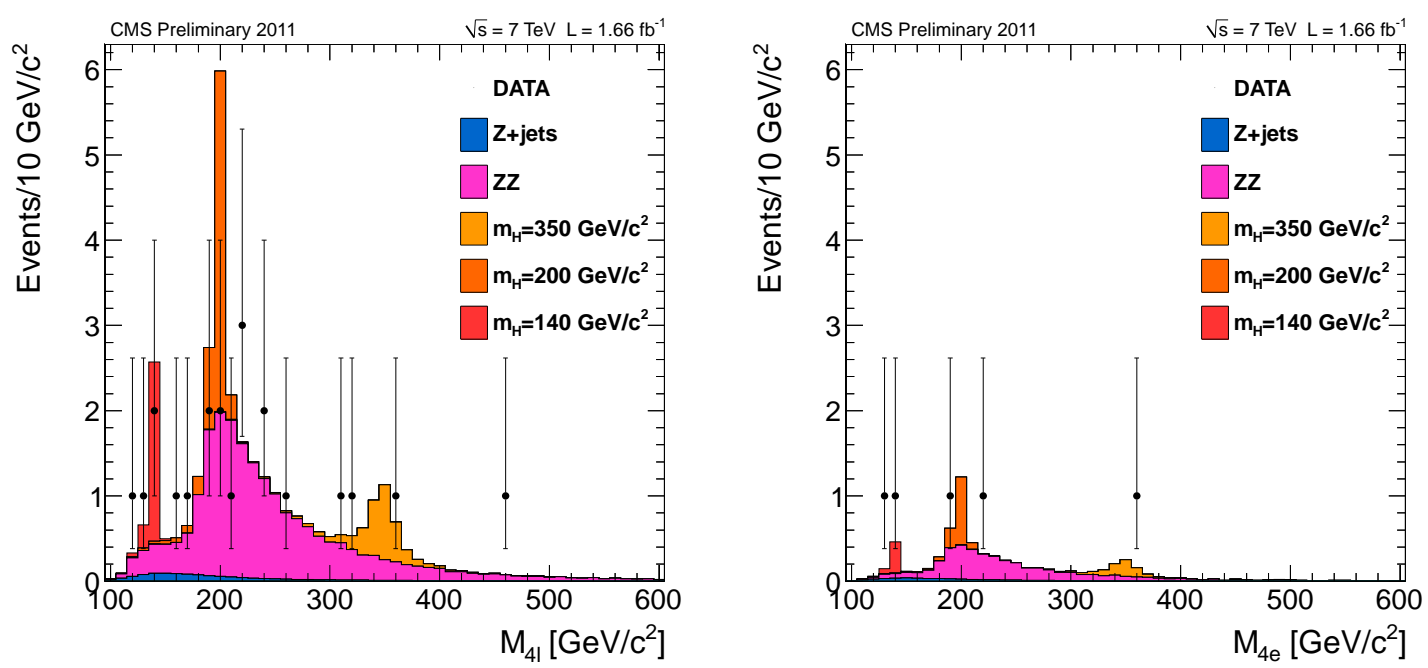

Figure 10: Observed invariant mass distribution of four leptons for all channels combined (left), and for 4e channel (right). Expected signal $\mathrm{H} \rightarrow \mathrm{ZZ}$ signal with $\mathrm{m}_{\mathrm{H}}=140,200$, and $300 \mathrm{GeV} / \mathrm{c}^{2}$ is shown.

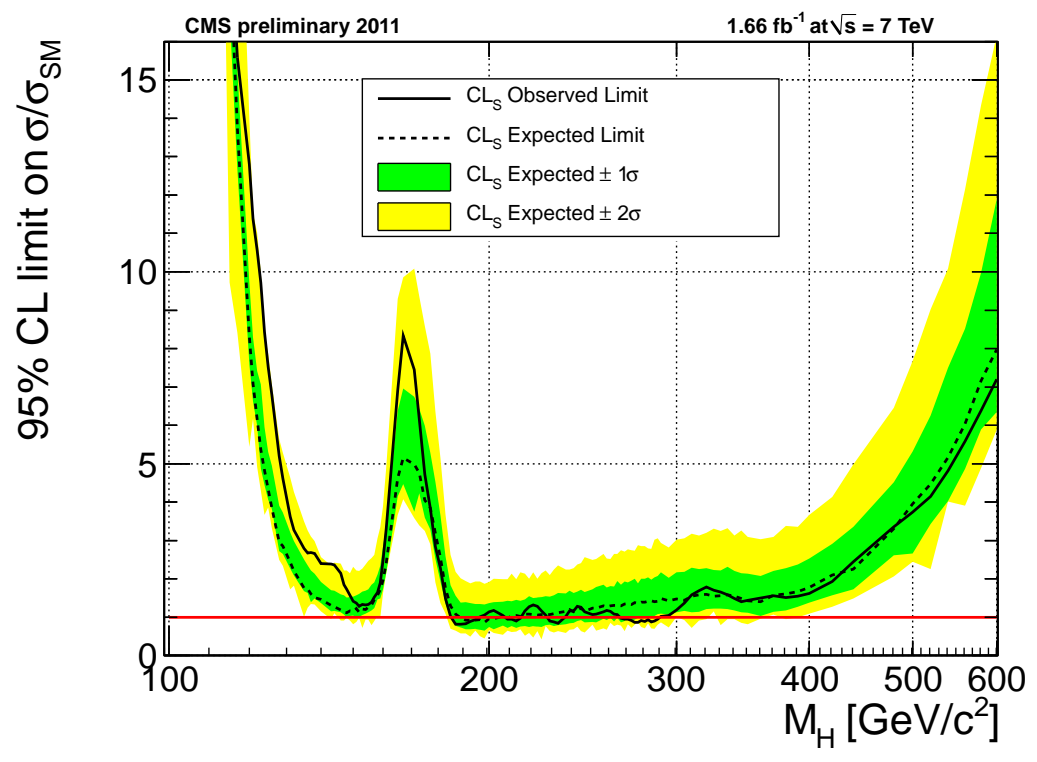

Figure 11: Observed 95\% CL exclusion limit for searches in the $\mathrm{H} \rightarrow \mathrm{ZZ} \rightarrow 11$ with with $1.66 \mathrm{fb}^{-1}$ of data collected at $\sqrt{\mathrm{s}}=7 \mathrm{TeV}$.

irreducible $\mathrm{ZZ}$ production, while the secondary background list includes $\mathrm{Z}+\mathrm{b} \overline{\mathrm{b}}, \mathrm{Z}+\mathrm{jets}, \mathrm{W}+\mathrm{jet}, \mathrm{t} \overline{\mathrm{t}}$ and QCD. Figure 10 presents invariant mass distribution of four leptons, combining all channels (left), and for 4e channel only (right).

Figure 11 presents the expected 95\% CL exclusion limit as a function of the Higgs mass. The observed exclusion limit at 95\% CL is about 1 to 2 times the Standard Model cross section, in the range $150<\mathrm{m}_{\mathrm{H}}<420 \mathrm{GeV} / \mathrm{c}^{2}$. 


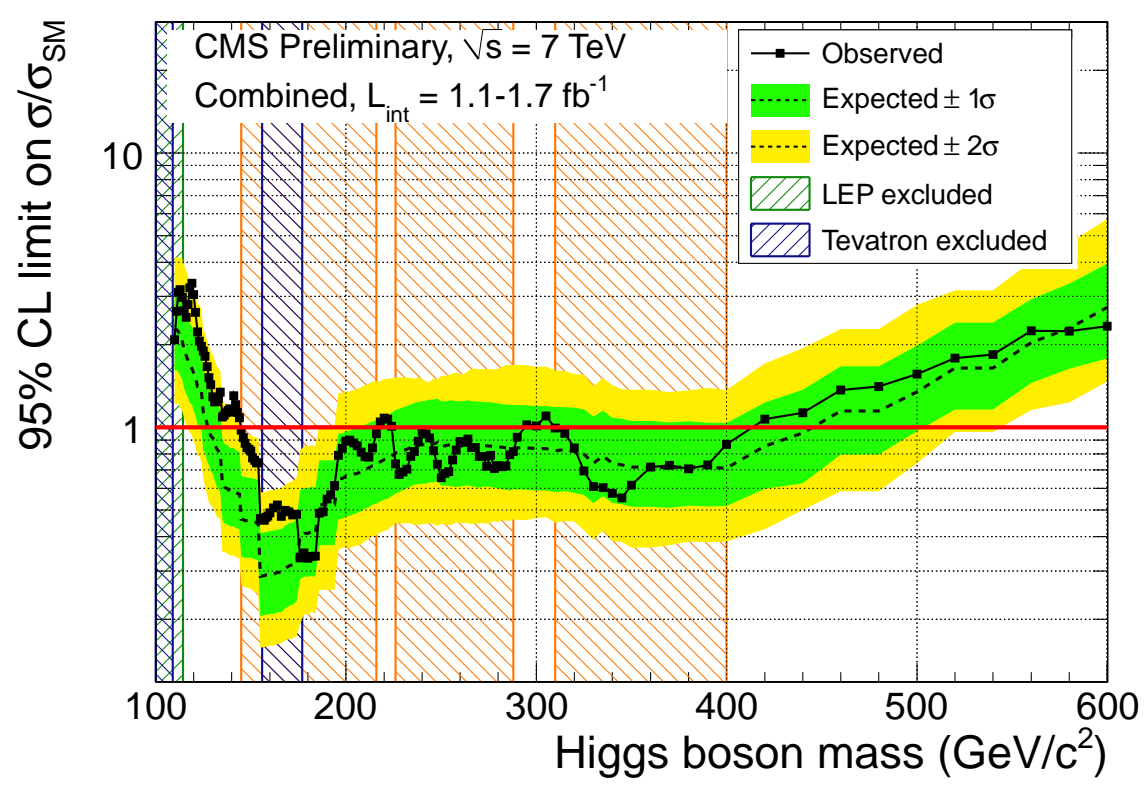

Figure 12: Observed 95\% CL exclusion limits for a SM Higgs search at $\sqrt{\mathrm{s}}=7$ with up to $1.7 \mathrm{fb}^{-1}$. The dashed line indicates the median expected limit on $\mathrm{m}$ for the background-only hypothesis, while the green/yellow bands indicate the ranges that are expected to contain $68 \% / 95 \%$ of all observed limit excursions from the median.

\section{Combination of the SM Higgs searches}

Figure 12 presents 95\% exclusion limits after combining all the SM Higgs boson channels analysed by the CMS Collaboration. With up to $1.7 \mathrm{fb}^{-1}$ of data analysed by the CMS Collaboration the SM Higgs boson is excluded at 95\% CL in three mass ranges: 145-216, 226-288, and $310-400 \mathrm{GeV} / \mathrm{c}^{2}$, while the expected exclusion in the absence of a signal is $130-440 \mathrm{GeV} / \mathrm{c}^{2}$. All differences between the expected and observed event yields are within the statistical errors, and the largest excursion of the observed data from the expected has a probability of 0.4 after taking into account the look-elsewhere effect.

\section{Conclusions}

The CMS experiment conducted Higgs boson searches in number of channels with up to $1.7 \mathrm{fb}^{-1}$ of data. No signal was observed in any of those, providing 95\% CL exclusion limits in ranges: $145-216,226-288$ and $310-400 \mathrm{GeV} / \mathrm{c}^{2}$. The Higgs boson searches at the CMS continues, as LHC provides more pp collisions in 2011 and 2012. It is expected that with the full set of data expected by the end of 2012 the full range of SM Higgs boson mass should be covered.

\section{Acknowledgments}

The author was supported by the HOMING PLUS programme of Foundation for Polish Science, cofinanced from European Union, Regional Development Fund. 


\section{References}

[1] ALEPH Collab., DELPHI Collab., L3 Collab., OPAL Collab. and The LEP Working Group for Higgs Boson Searches, Search for the Standard Model Higgs boson at LEP, Phys. Lett. B 565, 61 (2003).

[2] CDF Collab., D0 Collab., the Tevatron New Physics, Higgs Working Group, Combined CDF and DO Upper Limits on Standard Model Higgs Boson Production with up to $8.6 \mathrm{fb}^{-1}$ of Data, arXiv:1107.5518v2; FERMILAB-CONF-11-354-E, CERN, Geneva, 2011.

[3] CMS Collab., The CMS experiment at the CERN LHC, JINST 3, S08004 (2008).

[4] CMS Collab., Measurement of WW Production and Search for the Higgs Boson in pp Collisions at $\sqrt{s}=7 \mathrm{TeV}$, Phys. Lett. B 699, 25 (2011).

[5] CMS Collab., Search for Neutral MSSM Higgs Bosons Decaying to Tau Pairs in pp Collisions at $\sqrt{s}=7$ TeV, Phys. Rev. Lett. 106, 231801 (2011).

[6] LHC Higgs Cross Section Working Group (S. Dittmaier, C. Mariotti, G. Passarino, R. Tanaka (Eds.) et al.), Handbook of LHC Higgs Cross Sections: 1. Inclusive Observables, arXiv:1101.0593 [hep-ph]. 